Corrigendum

\title{
Corrigendum to "Enhancing ecosystem services in sustainable agriculture: Biofertilization and biofortification of chickpea (Cicer arietinum L.) by arbuscular mycorrhizal fungi."
}

\author{
Elisa Pellegrino ${ }^{\mathrm{a}, \mathrm{b}, *}$, Stefano Bedini ${ }^{\mathrm{b}}$ \\ ${ }^{a}$ Institute of Life Sciences, Scuola Superiore Sant'Anna, P.za Martiri della Libertà 33, 56127 Pisa, Italy \\ ${ }^{\mathrm{b}}$ Department of Agriculture, Food and Environment, University of Pisa, Via del Borghetto 80, 56124 Pisa, Italy
}

After publication it was brought to our attention that the experiment should have been described as a split-plot instead of a completely randomized design. Consequently, we have added more detailed information on the experimental setup and re-analyzed the data set according to the two-way split-plot design. Although there is no effect of this error on the discussion or conclusions of the paper, we have added the following corrections to the article. We apologize to the readers for this mistake.

On p. 431, left-hand column, lines 48-58 replace "A two-factor design.....weed-free." with:

Plots ( $5 \mathrm{~m} \times 3 \mathrm{~m} ; 1 \mathrm{~m}$ inter-plot space) were sown on October 2004 (autumn sowing) $(n=15)$ with $40 \times 10 \mathrm{~cm}$ row spacing and $10 \mathrm{~cm}$ border spacing. The total area of the experiment was about $900 \mathrm{~m}^{2}$. Plots were dug $(10 \mathrm{~cm} \mathrm{depth})$ and harrowed $(5 \mathrm{~cm}$ depth) and then inoculated with $5.3 \mathrm{~kg} \mathrm{plot}^{-1}$ of crude inoculum (for the AM fungal treatments) or heat killed inoculum (for the control) along the rows in September 2004. Three seeds were placed in each planting position and, after emergence, the seedlings were thinned to one. Due to a failure of about $50 \%$ of the chickpea germination after autumn sowing and since in Mediterranean areas the cultivation of chickpea takes place with both autumn and spring sowing, we decided to add a spring sowing treatment to the trial. Therefore, the plots were divided into two sub-plots $(5 \mathrm{~m} \times 1.5 \mathrm{~m})$, one representing winter sowing, where we kept the winter-sown seedlings, and the other representing the spring sowing. In the spring-sown sub-plots $(n=15)$, we eliminated the autumn-sown seedlings and in March 2005 we sowed the chickpea at the same spots where the autumn-sown seedlings failed. A two-factor split-plot design was applied with the inoculum treatment (IMA1, IMA5, FMix, LMix and the control) as main plot factor and the sowing time (autumn and spring sowing) as split-plot factor and three replicate plots.

On p. 431, right-hand column, lines 20-24, replace "Data were analyzed.....completely randomized design." with:

Data were analyzed by two-way split-plot (inoculum treatment and sowing time as main and split-plot factors, respectively) or one-way ANOVA (inoculum treatment as factor). Data were ln- and arcsine-transformed when needed to fulfil the assumptions of the ANOVA, which was carried out according to the split-plot design.

On p. 432, right-hand column, lines 45 , replace " $(P=0.738$ and $P=0.483$, respectively)" with: $(P=0.233$ and $P=0.642$, respectively).

Replace Tables 4 and 5 with the following ones:

Table 4

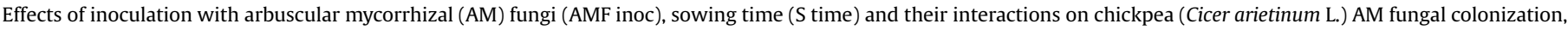
plant growth, yield and yield components at harvest.

\begin{tabular}{|c|c|c|c|c|}
\hline Factors $^{\mathrm{a}}$ & AM fungal colonization & Shoot dry matter ${ }^{b}$ & Collar diameter & Grain yield $^{\mathrm{C}}$ \\
\hline AMF inoc ${ }^{d}$ & $<0.001^{e}$ & 0.020 & 0.004 & 0.018 \\
\hline S time & 0.086 & 0.016 & $<0.001$ & 0.060 \\
\hline $\mathrm{AMF}$ inoc $\times \mathrm{S}$ time & 0.134 & 0.189 & 0.254 & 0.246 \\
\hline
\end{tabular}

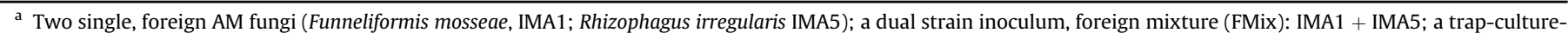
enriched locally-sourced AM fungal community (local mixture = LMix) and a mock inoculum as control; S time: autumn (October) and spring (March).

$\mathrm{b}$ Leaf and stem dry weight.

c Measurements per plant.

d Two-way split plot ANOVAs: AMF inoc, main plot factor; $\mathrm{S}$ time, split-plot factor.

e $P$-values of the two-way ANOVAs: in bold statistically significant values $(P \leq 0.05)$. Replicates field plots were three per treatment.

DOI of original article: http://dx.doi.org/10.1016/j.soilbio.2013.09.030.

* Corresponding author. Institute of Life Sciences, Scuola Superiore Sant'Anna, P.za Martiri della Libertà 33, 56127 Pisa, Italy.

E-mail address: epellegrino@hotmail.it (E. Pellegrino). 
Table 5

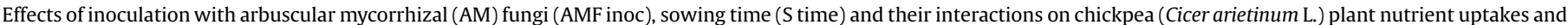
grain biofortification.

\begin{tabular}{|c|c|c|c|c|c|}
\hline \multirow[t]{2}{*}{ Factors $^{a}$} & \multicolumn{2}{|l|}{ Shoot } & \multicolumn{3}{|l|}{ Grain } \\
\hline & $\mathrm{N}^{\mathrm{b}}$ & $\mathrm{P}$ & $\mathrm{N}$ & $\mathrm{Fe}$ & $\mathrm{Zn}$ \\
\hline AMF inoc ${ }^{c}$ & $<\mathbf{0 . 0 0 1}^{\mathrm{d}}$ & 0.008 & 0.373 & $<0.001$ & $<0.001$ \\
\hline S time & 0.086 & 0.292 & 0.133 & $<0.001$ & 0.284 \\
\hline AMF inoc $\times S$ time & 0.174 & 0.300 & 0.540 & 0.807 & 0.772 \\
\hline
\end{tabular}

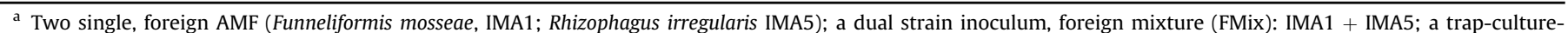
enriched locally-sourced AM fungal community (local mixture = LMix) and a mock inoculum as control; S time: autumn (October) and spring (March).

b Concentrations.

c Two-way split plot ANOVAs: AMF inoc, main plot factor; $\mathrm{S}$ time, split-plot factor.

d $P$-values of the two-way ANOVAs: in bold statistically significant values $(P \leq 0.05)$. Replicates field plots were three per treatment. 